\title{
On Weak Solutions of Stochastic Differential Equations
}

\author{
Martina Hofmanová, Jan Seidler
}

\begin{abstract}
A new proof of existence of weak solutions to stochastic differential equations with continuous coefficients based on ideas from infinite-dimensional stochastic analysis is presented. The proof is fairly elementary, in particular, neither theorems on representation of martingales by stochastic integrals nor results on almost sure representation for tight sequences of random variables are needed.
\end{abstract}

Keywords: stochastic differential equations, weak solutions, fractional integrals

Mathematics Subject Classification: $60 \mathrm{H} 10$

0. Introduction. In this paper, we provide a modified proof of Skorokhod's classical theorem on existence of (weak) solutions to a stochastic differential equation

$$
\mathrm{d} X=b(t, X) \mathrm{d} t+\sigma(t, X) \mathrm{d} W, \quad X(0)=\varphi,
$$

where $b:[0, T] \times \mathbb{R}^{m} \longrightarrow \mathbb{R}^{m}$ and $\sigma:[0, T] \times \mathbb{R}^{m} \longrightarrow \mathbb{M}_{m \times n}$ are Borel functions of at most linear growth continuous in the second variable. (Henceforward, by $\mathbb{M}_{m \times n}$ we shall denote the space of all $m$-by- $n$ matrices over $\mathbb{R}$ endowed with the HilbertSchmidt norm $\|A\|=\left(\operatorname{Tr} A A^{*}\right)^{1 / 2}$.) Our proof combines tools that were proposed for handling weak solutions of stochastic evolution equations in infinite-dimensional spaces, where traditional methods cease to work, with results on preservation of the local martingale property under convergence in law. In finite-dimensional situation, the "infinite-dimensional" methods simplify considerably and in our opinion the alternative proof based on them is more lucid and elementary than the standard one. A positive teaching experience of the second author was, in fact, the main motivation for writing this paper. Moreover, we believe that the reader may find the comparison with other available approaches illuminating.

To explain our argument more precisely, let us recall the structure of the usual proof; for notational simplicity, we shall consider (in the informal introduction only) autonomous equations. Kiyosi Itô showed in his seminal papers (see e.g. [9], [10]) that a stochastic differential equation

$$
\begin{gathered}
\mathrm{d} X=b(X) \mathrm{d} t+\sigma(X) \mathrm{d} W \\
X(0)=\varphi
\end{gathered}
$$

driven by an $n$-dimensional Wiener process $W$ has a unique solution provided that $b: \mathbb{R}^{m} \longrightarrow \mathbb{R}^{m}, \sigma: \mathbb{R}^{m} \longrightarrow \mathbb{M}_{m \times n}$ are Lipschitz continuous functions. A next important step was taken by A. Skorokhod ([16], [17]) in 1961, who proved that

This research was supported by the GA ČR Grant no. P201/10/0752. 
there exists a solution to $(0.1),(0.2)$ if $b$ and $\sigma$ are continuous functions of at most linear growth, i.e.

$$
\sup _{x \in \mathbb{R}^{m}} \frac{\|b(x)\|+\|\sigma(x)\|}{1+\|x\|}<\infty .
$$

It was realized only later that two different concepts of a solution are involved: for Lipschitzian coefficients, there exists an $\left(\mathscr{F}_{t}\right)$-progressively measurable process in $\mathbb{R}^{m}$ solving $(0.1)$ and such that $X(0)=\varphi$, whenever $\left(\Omega, \mathscr{F},\left(\mathscr{F}_{t}\right), \boldsymbol{P}\right)$ is a stochastic basis carrying an $n$-dimensional $\left(\mathscr{F}_{t}\right)$-Wiener process and $\varphi$ is an $\mathscr{F}_{0}$-measurable function. (We say that $(0.1),(0.2)$ has a strong solution.) On the other hand, for continuous coefficients, a stochastic basis $(\Omega, \mathscr{F},(\mathscr{F} t), \boldsymbol{P})$, an $n$-dimensional $\left(\mathscr{F}_{t}\right)$-Wiener process $W$ and an $\left(\mathscr{F}_{t}\right)$-progressively measurable process $X$ may be found such that $X$ solves $(0.1)$ and $X(0)$ and $\varphi$ have the same law. (We speak about existence of a weak solution to $(0.1),(0.2)$ in such a case.) It is well known that this difference is substantial in general: under assumptions of the Skorokhod theorem strong solutions need not exist (see [1]).

Skorokhod's existence theorem is remarkable not only by itself, but also because of the method of its proof. To present it, we need some notation: if $M$ and $N$ are continuous real local martingales, then by $\langle M\rangle$ we denote the quadratic variation of $M$ and by $\langle M, N\rangle$ the cross-variation of $M$ and $N$. Let $M=\left(M^{i}\right)_{i=1}^{m}$ and $N=$ $\left(N^{j}\right)_{j=1}^{n}$ be continuous local martingales with values in $\mathbb{R}^{m}$ and $\mathbb{R}^{n}$, respectively. By $\langle\langle M\rangle\rangle$ we denote the tensor quadratic variation of $M,\langle\langle M\rangle\rangle=\left(\left\langle M^{i}, M^{k}\right\rangle\right)_{i, k=1}^{m}$, and we set $\langle M\rangle=\operatorname{Tr}\langle\langle M\rangle\rangle$. Analogously, we define

$$
M \otimes N=\left(M^{i} N^{j}\right)_{i=1 j=1}^{m}, \quad\langle\langle M, N\rangle\rangle=\left(\left\langle M^{i}, N^{j}\right\rangle\right)_{i=1 j=1}^{m} n
$$

Let $X$ and $Y$ be random variables with values in the same measurable space $(E, \mathscr{E})$, we write $X \stackrel{\mathscr{D}}{\sim} Y$ if $X$ and $Y$ have the same law on $\mathscr{E}$. Similarly, $X \stackrel{\mathscr{D}}{\sim} \nu$ means that the law of $X$ is a probability measure $\nu$ on $\mathscr{E}$.

Let

$$
\mathrm{d} X_{r}=b_{r}\left(X_{r}\right) \mathrm{d} t+\sigma_{r}\left(X_{r}\right) \mathrm{d} W, \quad X_{r}(0)=\varphi
$$

be a sequence of equations which have strong solutions and approximate (0.1) in a suitable sense. (We shall approximate $b$ and $\sigma$ by Lipschitz continuous functions having the same growth as $b$ and $\sigma$, but likewise it is possible to use e.g. finite difference approximations.) The linear growth hypothesis makes it possible to prove that

$$
\text { the laws of }\left\{X_{r} ; r \geq 1\right\} \text { are tight, }
$$

that is, form a relatively weakly compact set of measures on the space of continuous trajectories. Then Skorokhod's theorem on almost surely converging realizations of converging laws (see e.g. [5], Theorem 11.7.2) may be invoked, which yields a 
subsequence $\left\{X_{r_{k}}\right\}$ of $\left\{X_{r}\right\}$, a probability space $(\tilde{\Omega}, \tilde{\mathscr{F}}, \tilde{\boldsymbol{P}})$ and sequences $\left\{\tilde{X}_{k} ; k \geq\right.$ $0\},\left\{\tilde{W}_{k} ; k \geq 0\right\}$ such that

$$
\left(X_{r_{k}}, W\right) \stackrel{\mathscr{D}}{\sim}\left(\tilde{X}_{k}, \tilde{W}_{k}\right), k \geq 1 ; \quad\left(\tilde{X}_{k}, \tilde{W}_{k}\right) \underset{k \rightarrow \infty}{\stackrel{\tilde{\boldsymbol{P}}_{\text {-a.s. }}}{\longrightarrow}}\left(\tilde{X}_{0}, \tilde{W}_{0}\right)
$$

It is claimed that $\tilde{X}_{0}$ is the (weak) solution looked for. Skorokhod's papers [16] and [17] are written in a very concise way and details of proofs are not offered; nowadays standard version of Skorokhod's proof is as follows (see [18], Theorem 6.1.6, [8], Theorem IV.2.2, [12], Theorem 5.4.22): under a suitable integrability assumption upon the initial condition,

$$
M_{k}=X_{r_{k}}-X_{r_{k}}(0)-\int_{0}^{\cdot} b_{r_{k}}\left(X_{r_{k}}(s)\right) \mathrm{d} s
$$

is a martingale with a (tensor) quadratic variation

$$
\left\langle\left\langle M_{k}\right\rangle\right\rangle=\int_{0}^{\cdot} \sigma_{r_{k}}\left(X_{r_{k}}(s)\right) \sigma_{r_{k}}^{*}\left(X_{r_{k}}(s)\right) \mathrm{d} s,
$$

for all $k \geq 1$. Equality in law (0.4) implies that also

$$
\tilde{M}_{k}=\tilde{X}_{k}-\tilde{X}_{k}(0)-\int_{0} b_{r_{k}}\left(\tilde{X}_{k}(s)\right) \mathrm{d} s
$$

are martingales for $k \geq 1$, with quadratic variations

$$
\left\langle\left\langle\tilde{M}_{k}\right\rangle\right\rangle=\int_{0}^{\cdot} \sigma_{r_{k}}\left(\tilde{X}_{k}(s)\right) \sigma_{r_{k}}^{*}\left(\tilde{X}_{k}(s)\right) \mathrm{d} s .
$$

Using convergence $\tilde{\boldsymbol{P}}$-almost everywhere, it is possible to show that

$$
\tilde{M}_{0}=\tilde{X}_{0}-\tilde{X}_{0}(0)-\int_{0} b\left(\tilde{X}_{0}(s)\right) \mathrm{d} s
$$

is a martingale with a quadratic variation

$$
\left\langle\left\langle\tilde{M}_{0}\right\rangle\right\rangle=\int_{0}^{\cdot} \sigma\left(\tilde{X}_{0}(s)\right) \sigma^{*}\left(\tilde{X}_{0}(s)\right) \mathrm{d} s .
$$

By the integral representation theorem for martingales with an absolutely continuous quadratic variation (see e.g. [12], Theorem 3.4.2, or [8], Theorem II.7.1'), there exists a Wiener process $\hat{W}$ (on an extended probability space) satisfying

$$
\tilde{M}_{0}=\int_{0}^{\cdot} \sigma\left(\tilde{X}_{0}(s)\right) \mathrm{d} \hat{W}(s) .
$$


Therefore, $\left(\hat{W}, \tilde{X}_{0}\right)$ is a weak solution to $(0.1),(0.2)$. (In the cited books, martingale problems are used instead of weak solutions. Then the integral representation theorem is hidden in the construction of a weak solution from a solution to the martingale problem, so a complete proof is essentially the one sketched above.)

This procedure has two rather nontrivial inputs: the Skorokhod representation theorem, and the integral representation theorem whose proof, albeit based on a simple and beautiful idea, becomes quite technical if the space dimension is greater than one. An alternative approach to identification of the limit was discovered recently (see [3], [14]) in the course of study of stochastic wave maps between manifolds, where integral representation theorems for martingales are no longer available. The new method, which refers only to basic properties of martingales and stochastic integrals, may be described in the case of the problem $(0.1),(0.2)$ in the following way: One starts again with a sequence $\left\{\left(\tilde{X}_{k}, \tilde{W}_{k}\right)\right\}$ such that $(0.4)$ holds true. If the initial condition is $p$-integrable for some $p>2$, it can be shown in a straightforward manner, using the almost sure convergence, that

$$
\tilde{M}_{0}, \quad\left\|\tilde{M}_{0}\right\|^{2}-\int_{0}^{\cdot}\left\|\sigma\left(\tilde{X}_{0}(s)\right)\right\|^{2} \mathrm{~d} s, \quad \tilde{M}_{0} \otimes \tilde{W}_{0}-\int_{0}^{\cdot} \sigma\left(\tilde{X}_{0}(s)\right) \mathrm{d} s
$$

are martingales, in other words,

$$
\left\langle\tilde{M}_{0}-\int_{0}^{\cdot} \sigma\left(\tilde{X}_{0}(s)\right) \mathrm{d} \tilde{W}_{0}(s)\right\rangle=0
$$

whence one concludes that $\left(\tilde{W}_{0}, \tilde{X}_{0}\right)$ is a weak solution. If the additional integrability hypothesis on $\varphi$ is not satisfied, the proof remains almost the same, only a suitable cut-off procedure must be amended.

We take a step further and eliminate also the Skorokhod representation theorem. Let $\tilde{\boldsymbol{P}}_{k}$ be the laws of $\left(X_{r_{k}}, W\right)$ on the space $U=\mathscr{C}\left([0, T] ; \mathbb{R}^{m}\right) \times \mathscr{C}\left([0, T] ; \mathbb{R}^{n}\right)$; we know that the sequence $\left\{\tilde{\boldsymbol{P}}_{k}\right\}$ converges weakly to some measure $\tilde{\boldsymbol{P}}_{0}$. Denote by $(Y, B)$ the canonical process on $U$ and set

$$
\bar{M}_{k}=Y-Y(0)-\int_{0}^{\cdot} b_{r_{k}}(Y(s)) \mathrm{d} s, \quad k \geq 0
$$

$\left(\right.$ with $\left.b_{r_{0}}=b, \sigma_{r_{0}}=\sigma\right)$. Then

$$
\bar{M}_{k}, \quad\left\|\bar{M}_{k}\right\|^{2}-\int_{0}^{\cdot}\left\|\sigma_{r_{k}}(Y(s))\right\|^{2} \mathrm{~d} s, \quad \bar{M}_{k} \otimes B-\int_{0}^{\cdot} \sigma_{r_{k}}(Y(s)) \mathrm{d} s,
$$

are local martingales under the measure $\tilde{\boldsymbol{P}}_{k}$ for every $k \geq 1$, as can be inferred quite easily from the definition of the measure $\tilde{\boldsymbol{P}}_{k}$. Now one may try to use Theorem 
IX.1.17 from [11] stating, roughly speaking, that a limit in law of a sequence of continuous local martingales is a local martingale. We do not use this theorem explicitly, since to establish convergence in law of the processes $(0.5)$ as $k \rightarrow \infty$ is not simpler than to check the local martingale property for $k=0$ directly, but our argument is inspired by the proofs in the book [11]. The proof we propose is not difficult and it is almost self-contained, it requires only two auxiliary lemmas (with simple proofs) from [11] on continuity properties of certain first entrance times which we recall in Appendix. Once we know that the processes (0.5) are local martingales for $k=0$ as well, the trick from [3] and [14] may be used yielding that $(B, Y)$ is a weak solution to $(0.1),(0.2)$. It is worth mentioning that this procedure is independent of any integrability hypothesis on $\varphi$.

The proof of (0.3) not being our main concern notwithstanding, we decided to include a less standard proof of tightness inspired also by the theory of stochastic partial differential equations. We adopt an argument proposed by D. Gatarek and B. Gołdys in [6] (cf. also [4], Chapter 8), who introduced it when studying weak solutions to stochastic evolution equations in Hilbert spaces, and which relies on the factorization method of G. Da Prato, S. Kwapień and J. Zabczyk (see [4], Chapters 5 and 7 , for a thorough exposition) and on compactness properties of fractional integral operators. The fractional calculus has become popular amongst probabilists recently because of its applications to fractional Brownian motion driven stochastic integrals and a proof of tightness using it may suit some readers more than the traditional one based on estimates of moduli of continuity.

Let us close this Introduction by stating the result to be proved precisely.

Theorem 0.1. Let $b:[0, T] \times \mathbb{R}^{m} \longrightarrow \mathbb{R}^{m}$ and $\sigma:[0, T] \times \mathbb{R}^{m} \longrightarrow \mathbb{M}_{m \times n}$ be Borel functions such that $b(t, \cdot)$ and $\sigma(t, \cdot)$ are continuous on $\mathbb{R}^{m}$ for any $t \in[0, T]$ and the linear growth hypothesis is satisfied, that is

$$
\exists K_{*}<\infty \forall t \in[0, T] \forall x \in \mathbb{R}^{m} \quad\|b(t, x)\| \vee\|\sigma(t, x)\| \leq K_{*}(1+\|x\|) .
$$

Let $\nu$ be a Borel probability measure on $\mathbb{R}^{m}$. Then there exists a weak solution to the problem

$$
\mathrm{d} X=b(t, X) \mathrm{d} t+\sigma(t, X) \mathrm{d} W, \quad X(0) \stackrel{\mathscr{D}}{\sim} \nu
$$

We recall that a weak solution to $(0.7)$ is a triple $\left(\left(G, \mathscr{G}_{,}\left(\mathscr{G}_{t}\right), \boldsymbol{Q}\right), W, X\right)$, where $\left(G, \mathscr{G},\left(\mathscr{G}_{t}\right), \boldsymbol{Q}\right)$ is a stochastic basis with a filtration $\left(\mathscr{G}_{t}\right)$ that satisfies the usual conditions, $W$ is an $n$-dimensional $\left(\mathscr{G}_{t}\right)$-Wiener process and $X$ is an $\mathbb{R}^{m}$-valued $\left(\mathscr{G}_{t}\right)$-progressively measurable process such that $\boldsymbol{Q} \circ X(0)^{-1}=\nu$ and

$$
X(t)=X(0)+\int_{0}^{t} b(r, X(r)) \mathrm{d} r+\int_{0}^{t} \sigma(r, X(r)) \mathrm{d} W(r)
$$

for all $t \in[0, T] \boldsymbol{Q}$-almost surely. 
The rest of the paper is devoted to the proof of Theorem 0.1. In Section 1, a sequence of equations with Lipschitzian coefficients approximation (0.7) is constructed, tightness of the set of their solutions being shown in Section 2. In Section 3 , cluster points of the set of approximating solutions are identified as weak solutions to (0.7).

Acknowledgements. The authors are indebted to Martin Ondreját for many useful discussions.

1. Approximations. In this Section we introduce a sequence of equations which have strong solutions and approximate the problem (0.7). If $E$ and $F$ are metric spaces, we denote by $\mathscr{C}(E ; F)$ the space of all continuous mappings from $E$ to $F$. For brevity, we shall sometimes write $\mathscr{C}_{V}$ instead of $\mathscr{C}\left([0, T] ; \mathbb{R}^{V}\right)$ if $V \in \mathbb{N}$. If $f \in \mathscr{C}([0, T] ; F)$ and $s \in[0, T]$ then the restriction of $f$ to the interval $[0, s]$ will be denoted by $\varrho_{s} f$. Plainly, $\varrho_{s}: \mathscr{C}([0, T] ; F) \longrightarrow \mathscr{C}([0, s] ; F)$ is a continuous mapping. Finally, $L^{q}\left(G ; \mathbb{R}^{V}\right)$ stands for the space of $q$-integrable functions on $G$ with values in $\mathbb{R}^{V}$.

Our construction is based on the following proposition.

Proposition 1.1. Suppose that $F: \mathbb{R}_{+} \times \mathbb{R}^{N} \longrightarrow \mathbb{R}^{V}$ is a Borel function of at most linear growth, i.e.

$$
\exists L<\infty \forall t \geq 0 \forall x \in \mathbb{R}^{N} \quad\|F(t, x)\| \leq L(1+\|x\|),
$$

such that $F(t, \cdot) \in \mathscr{C}\left(\mathbb{R}^{N} ; \mathbb{R}^{V}\right)$ for any $t \in \mathbb{R}_{+}$. Then there exists a sequence of Borel functions $F_{k}: \mathbb{R}_{+} \times \mathbb{R}^{N} \longrightarrow \mathbb{R}^{V}, k \geq 1$, which have at most linear growth uniformly in $k$, namely

$$
\forall k \geq 1 \forall t \geq 0 \forall x \in \mathbb{R}^{N} \quad\left\|F_{k}(t, x)\right\| \leq L(2+\|x\|),
$$

which are Lipschitz continuous in the second variable uniformly in the first one,

$$
\forall k \geq 1 \exists L_{k}<\infty \forall t \geq 0 \forall x, y \in \mathbb{R}^{N} \quad\left\|F_{k}(t, x)-F_{k}(t, y)\right\| \leq L_{k}\|x-y\|,
$$

and which satisfy

$$
\lim _{k \rightarrow \infty} F_{k}(t, \cdot)=F(t, \cdot) \quad \text { locally uniformly on } \mathbb{R}^{N}
$$

for all $t \geq 0$.

The proof is rather standard so it is not necessary to dwell on its details: one takes a smooth function $\zeta \in \mathscr{C}^{\infty}\left(\mathbb{R}^{N}\right)$ such that $\zeta \geq 0, \operatorname{supp} \zeta \subseteq\left\{x \in \mathbb{R}^{N} ;\|x\| \leq 1\right\}$ and $\int_{\mathbb{R}^{N}} \zeta \mathrm{d} x=1$ and sets

$$
G_{k}(t, x)=k^{N} \int_{\mathbb{R}^{N}} F(t, y) \zeta(k(x-y)) \mathrm{d} y
$$


for $k \geq 1, t \geq 0$ and $x \in \mathbb{R}^{N}$. The functions $G_{k}$ have all desired properties except for being only locally Lipschitz, but it is possible to modify them outside a sufficiently large ball in an obvious manner.

Let the coefficients $b$ and $\sigma$ satisfy the assumptions of Theorem 0.1. Using Proposition 1.1 we find Borel functions $b_{k}:[0, T] \times \mathbb{R}^{m} \longrightarrow \mathbb{R}^{m}$ and $\sigma_{k}:[0, T] \times$ $\mathbb{R}^{m} \longrightarrow \mathbb{M}_{m \times n}, k \geq 1$, such that

$$
\sup _{k \geq 1} \sup _{t \in[0, T]}\left\{\left\|b_{k}(t, x)\right\| \vee\left\|\sigma_{k}(t, x)\right\|\right\} \leq K_{*}(2+\|x\|), \quad x \in \mathbb{R}^{m}
$$

$b_{k}(t, \cdot)$ and $\sigma_{k}(t, \cdot)$ are Lipschitz continuous uniformly in $t \in[0, T]$ and converge locally uniformly on $\mathbb{R}^{m}$ as $k \rightarrow \infty$ to $b(t, \cdot)$ and $\sigma(t, \cdot)$, respectively, for all $t \in[0, T]$. Fix an arbitrary stochastic basis $\left(\Omega, \mathscr{F},\left(\mathscr{F}_{t}\right), \boldsymbol{P}\right)$, on which an $n$-dimensional $\left(\mathscr{F}_{t}\right)$ Wiener process $W$ and an $\mathscr{F}_{0}$-measurable random variable $\varphi: \Omega \longrightarrow \mathbb{R}^{m}$ with $\varphi \stackrel{\mathscr{D}}{\sim} \nu$ are defined. It is well known that for any $k \geq 1$ there exists a unique $\left(\mathscr{F}_{t}\right)$ progressively measurable $\mathbb{R}^{m}$-valued stochastic process $X_{k}$ solving the equation

$$
\mathrm{d} X_{k}=b_{k}\left(t, X_{k}\right) \mathrm{d} t+\sigma_{k}\left(t, X_{k}\right) \mathrm{d} W, \quad X_{k}(0)=\varphi .
$$

Moreover, for any $p \in\left[2, \infty\left[\right.\right.$ there exists a constant $C_{*}<\infty$, depending only on $p$, $T$ and $K_{*}$, such that

$$
\sup _{k \geq 1} \boldsymbol{E} \sup _{0 \leq t \leq T}\left\|X_{k}(t)\right\|^{p} \leq C_{*}\left(1+\boldsymbol{E}\|\varphi\|^{p}\right)
$$

provided that

$$
\int_{\mathbb{R}^{m}}\|x\|^{p} \mathrm{~d} \nu(x)=\boldsymbol{E}\|\varphi\|^{p}<\infty .
$$

2. Tightness. Let $\left\{X_{k} ; k \geq 1\right\}$ be the sequence of solutions to (1.2). Plainly, the processes $X_{k}$ may be viewed as random variables $X_{k}: \Omega \longrightarrow \mathscr{C}_{m}$ (where the Polish metric space $\mathscr{C}_{m}$ is endowed with its Borel $\sigma$-algebra). In this section, we aim at establishing the following proposition.

Proposition 2.1. The set $\left\{\boldsymbol{P} \circ X_{k}^{-1} ; k \geq 1\right\}$ of Borel probability measures on $\mathscr{C}\left([0, T] ; \mathbb{R}^{m}\right)$ is tight.

To this end, let us recall the definition of the Riemann-Liouville (or fractional integral) operator: if $\left.q \in] 1, \infty], \alpha \in] \frac{1}{q}, 1\right]$ and $f \in L^{q}\left([0, T] ; \mathbb{R}^{m}\right)$, we define a function $R_{\alpha} f:[0, T] \longrightarrow \mathbb{R}^{m}$ by

$$
\left(R_{\alpha} f\right)(t)=\int_{0}^{t}(t-s)^{\alpha-1} f(s) \mathrm{d} s, \quad 0 \leq t \leq T
$$


The definition is correct, as an easy application of the Hölder inequality shows. Note that, in particular, $R_{1} f=\int_{0}^{\cdot} f(t) \mathrm{d} t$. It is well-known (and may be checked by very straightforward calculations) that $R_{\alpha}$ is a bounded linear operator from $L^{q}\left([0, T] ; \mathbb{R}^{m}\right)$ to the space $\mathscr{C}^{0, \alpha-1 / q}\left([0, T] ; \mathbb{R}^{m}\right)$ of $\left(\alpha-\frac{1}{q}\right)$-Hölder continuous functions (see e.g. [15], Theorem 3.6). Balls in $\mathscr{C}^{0, \alpha-1 / q}\left([0, T] ; \mathbb{R}^{m}\right)$ are relatively compact in $\mathscr{C}\left([0, T] ; \mathbb{R}^{m}\right)$ by the Arzelà-Ascoli theorem, hence we arrive at

Lemma 2.2. If $q \in] 1, \infty]$ and $\left.\alpha \in] \frac{1}{q}, 1\right]$, then $R_{\alpha}$ is a compact linear operator from $L^{q}\left([0, T] ; \mathbb{R}^{m}\right)$ to $\mathscr{C}\left([0, T] ; \mathbb{R}^{m}\right)$.

We shall need also a Fubini-type theorem for stochastic integrals in the following form (a more general result may be found in [4], Theorem 4.18):

Lemma 2.3. Let $(X, \Sigma, \mu)$ be a finite measure space, $\left(G, \mathscr{G},\left(\mathscr{G}_{t}\right), \boldsymbol{Q}\right)$ a stochastic basis, and $B$ an n-dimensional $\left(\mathscr{G}_{t}\right)$-Wiener process. Denote by $\mathscr{M}$ the $\sigma$-algebra of $\left(\mathscr{G}_{t}\right)$-progressively measurable sets and assume that $\psi:[0, T] \times G \times X \longrightarrow \mathbb{M}_{m \times n}$ is an $\mathscr{M} \otimes \Sigma$-measurable mapping such that

$$
\int_{X}\left(\int_{0}^{T} \int_{G}\|\psi(s, x)\|^{2} \mathrm{~d} \boldsymbol{Q} \mathrm{d} s\right)^{1 / 2} \mathrm{~d} \mu(x)<\infty .
$$

Then

$$
\int_{X}\left[\int_{0}^{T} \psi(s, x) \mathrm{d} B(s)\right] \mathrm{d} \mu(x)=\int_{0}^{T}\left[\int_{X} \psi(s, x) \mathrm{d} \mu(x)\right] \mathrm{d} B(s)
$$

$Q$-almost surely.

The last auxiliary result to be recalled is the Young inequality for convolutions (see, for example, [13], Theorem 4.2).

Lemma 2.4. Let $p, r, s \in[1, \infty]$ satisfy

$$
\frac{1}{p}+\frac{1}{q}=1+\frac{1}{s}
$$

If $f \in L^{p}\left(\mathbb{R}^{d}\right)$ and $g \in L^{q}\left(\mathbb{R}^{d}\right)$, then the integral

$$
(f * g)(x) \equiv \int_{\mathbb{R}^{d}} f(x-y) g(y) \mathrm{d} y
$$

converges for almost all $x \in \mathbb{R}^{d}, f * g \in L^{s}\left(\mathbb{R}^{d}\right)$ and

$$
\|f * g\|_{L^{s}} \leq\|f\|_{L^{p}}\|g\|_{L^{q}}
$$


In fact, we shall need only a particular one-dimensional case of Lemma 2.4: if $f \in L^{p}(0, T), g \in L^{q}(0, T), \frac{1}{p}+\frac{1}{q}=1+\frac{1}{s}$, then

$$
\int_{0}^{T}\left|\int_{0}^{t} f(t-r) g(r) \mathrm{d} r\right|^{s} \mathrm{~d} t \leq\|f\|_{L^{p}(0, T)}^{s}\|g\|_{L^{q}(0, T)}^{S} .
$$

Now we derive a representation formula that plays a key role in our proof of Proposition 2.1.

Lemma 2.5. Let $\psi$ be an $\mathbb{M}_{m \times n}$-valued progressively measurable process such that

$$
\boldsymbol{E} \int_{0}^{T}\|\psi(s)\|^{q} \mathrm{~d} s<\infty
$$

for some $q>2$. Choose $\alpha \in] \frac{1}{q}, \frac{1}{2}[$ and set

$$
Z(t)=\int_{0}^{t}(t-u)^{-\alpha} \psi(u) \mathrm{d} W(u), \quad 0 \leq t \leq T
$$

Then

$$
\int_{0}^{t} \psi(s) \mathrm{d} W(s)=\frac{\sin \pi \alpha}{\pi}\left(R_{\alpha} Z\right)(t)
$$

for all $t \in[0, T] \boldsymbol{P}$-almost surely.

Proof. The result is well-known and widely used for infinite-dimensional systems (see e.g. [4], §5.3). For finite-dimensional equations, the proof is slightly simpler and thus it is repeated here for the reader's convenience.

Since $s^{-2 \alpha} \in L^{1}(0, T), \boldsymbol{E}\|\psi(\cdot)\|^{2} \in L^{1}(0, T)$, their convolution

$$
t \longmapsto \int_{0}^{t}(t-s)^{-2 \alpha} \boldsymbol{E}\|\psi(s)\|^{2} \mathrm{~d} s=\boldsymbol{E} \int_{0}^{t}\left|(t-s)^{-\alpha}\|\psi(s)\|\right|^{2} \mathrm{~d} s
$$

belongs to $L^{1}(0, T)$ as well and so is finite almost everywhere in $[0, T]$, which implies that $Z(t)$ is well defined for almost all $t \in[0, T]$. By the Burkholder-Davis-Gundy inequality,

$$
\begin{aligned}
\boldsymbol{E} \int_{0}^{T}\|Z(t)\|^{q} \mathrm{~d} t & =\int_{0}^{T} \boldsymbol{E}\left\|\int_{0}^{s}(s-u)^{-\alpha} \psi(u) \mathrm{d} W(u)\right\|^{q} \mathrm{~d} s \\
& \leq C_{q} \boldsymbol{E} \int_{0}^{T}\left(\int_{0}^{s}(s-u)^{-2 \alpha}\|\psi(u)\|^{2} \mathrm{~d} u\right)^{q / 2} \mathrm{~d} s \\
& \leq C_{q}\left(\int_{0}^{T} s^{-2 \alpha} \mathrm{d} s\right)^{q / 2}\left(\int_{0}^{T} \boldsymbol{E}\|\psi(u)\|^{q} \mathrm{~d} u\right)
\end{aligned}
$$


the last estimate being a consequence of (2.2) and the fact that $\boldsymbol{E}\|\psi(\cdot)\|^{2} \in$ $L^{q / 2}(0, T)$. Hence $Z(\cdot, \omega) \in L^{q}\left(0, T ; \mathbb{R}^{m}\right)$ for $\boldsymbol{P}$-almost all $\omega \in \Omega$ and $R_{\alpha} Z$ is well defined $\boldsymbol{P}$-almost surely.

Further,

$$
\begin{aligned}
\int_{0}^{t}\left(\boldsymbol{E} \int_{0}^{t}\right. & \left.\left\|(t-s)^{\alpha-1} \mathbf{1}_{[0, s[}(u)(s-u)^{-\alpha} \psi(u)\right\|^{2} \mathrm{~d} u\right)^{1 / 2} \mathrm{~d} s \\
& =\int_{0}^{t}(t-s)^{\alpha-1}\left(\int_{0}^{s}(s-u)^{-2 \alpha} \boldsymbol{E}\|\psi(u)\|^{2} \mathrm{~d} u\right)^{1 / 2} \mathrm{~d} s \\
& \leq\left(\int_{0}^{t} s^{(\alpha-1) q^{*}} \mathrm{~d} s\right)^{1 / q^{*}}\left(\int_{0}^{t}\left(\int_{0}^{s}(s-u)^{-2 \alpha} \boldsymbol{E}\|\psi(u)\|^{2} \mathrm{~d} u\right)^{q / 2} \mathrm{~d} s\right)^{1 / q} \\
& \leq\left(\int_{0}^{t} s^{(\alpha-1) q^{*}} \mathrm{~d} s\right)^{1 / q^{*}}\left(\int_{0}^{t} s^{-2 \alpha} \mathrm{d} s\right)^{1 / 2}\left(\int_{0}^{t} \boldsymbol{E}\|\psi(u)\|^{q} \mathrm{~d} u\right)^{1 / q}<\infty
\end{aligned}
$$

where $\frac{1}{q^{*}}+\frac{1}{q}=1$ and the Hölder and Young inequalities were used consecutively. This means that the hypothesis (2.1) of Lemma 2.3 is satisfied and this lemma may be used to obtain

$$
\begin{aligned}
\left(R_{\alpha} Z\right)(t) & =\int_{0}^{t}(t-s)^{\alpha-1}\left(\int_{0}^{s}(s-u)^{-\alpha} \psi(u) \mathrm{d} W(u)\right) \mathrm{d} s \\
& =\int_{0}^{t} \int_{0}^{t}(t-s)^{\alpha-1} \mathbf{1}_{[0, s[}(u)(s-u)^{-\alpha} \psi(u) \mathrm{d} W(u) \mathrm{d} s \\
& =\int_{0}^{t}\left[\int_{0}^{t}(t-s)^{\alpha-1} \mathbf{1}_{[0, s[}(u)(s-u)^{-\alpha} \mathrm{d} s\right] \psi(u) \mathrm{d} W(u) \\
& =\int_{0}^{t}\left[\int_{u}^{t}(t-s)^{\alpha-1}(s-u)^{-\alpha} \mathrm{d} s\right] \psi(u) \mathrm{d} W(u) \\
& =\int_{0}^{t} \underbrace{\left[\int_{0}^{1}(1-v)^{\alpha-1} v^{-\alpha} \mathrm{d} v\right]}_{=\frac{\pi}{\sin \pi \alpha}} \psi(u) \mathrm{d} W(u) .
\end{aligned}
$$

Q.E.D.

Proof of Proposition 2.1. Let an arbitrary $\varepsilon>0$ be given, we have to find a relatively compact set $K \subseteq \mathscr{C}_{m}$ such that

$$
\inf _{k \geq 1} \boldsymbol{P}\left\{X_{k} \in K\right\} \geq 1-\varepsilon
$$

In what follows, we shall denote by $D_{i}$ constants independent of $k$ and by $|\cdot|_{q}$ the norm of $L^{q}\left(0, T ; \mathbb{R}^{m}\right)$. 
First, we prove our claim under an additional assumption that there exists $p>2$ such that

$$
\boldsymbol{E}\|\varphi\|^{p}<\infty
$$

Plainly, a compact set $\Gamma \subseteq \mathbb{R}^{m}$ may be found satisfying

$$
\nu(\Gamma)=\boldsymbol{P}\{\varphi \in \Gamma\} \geq 1-\frac{\varepsilon}{3} .
$$

Take an $\alpha \in] \frac{1}{p}, \frac{1}{2}[$. By Lemma 2.5,

$$
\begin{aligned}
X_{k}(t) & =\varphi+\int_{0}^{t} b_{k}\left(s, X_{k}(s)\right) \mathrm{d} s+\int_{0}^{t} \sigma_{k}\left(s, X_{k}(s)\right) \mathrm{d} W(s) \\
& =\varphi+\left[R_{1} b\left(\cdot, X_{k}(\cdot)\right)\right](t)+\frac{\sin \pi \alpha}{\pi}\left(R_{\alpha} Z_{k}\right)(t), \quad 0 \leq t \leq T,
\end{aligned}
$$

$\boldsymbol{P}$-almost surely, where

$$
Z_{k}(s)=\int_{0}^{s}(s-u)^{-\alpha} \sigma_{k}\left(u, X_{k}(u)\right) \mathrm{d} W(u), \quad 0 \leq s \leq T
$$

Applying the Chebyshev inequality, (1.1) and (1.3) we get

$$
\begin{aligned}
\boldsymbol{P}\left\{\left|b_{k}\left(\cdot, X_{k}(\cdot)\right)\right|_{p} \geq \Lambda\right\} & \leq \frac{1}{\Lambda^{p}} \boldsymbol{E} \int_{0}^{T}\left\|b_{k}\left(t, X_{k}(t)\right)\right\|^{p} \mathrm{~d} t \\
& \leq \frac{1}{\Lambda^{p}} K_{*}^{p} \boldsymbol{E} \int_{0}^{T}\left(2+\left\|X_{k}(t)\right\|\right)^{p} \mathrm{~d} t \\
& \leq \frac{D_{1}}{\Lambda^{p}}\left(1+\boldsymbol{E}\|\varphi\|^{p}\right) .
\end{aligned}
$$

Similarly, invoking in addition the Burkholder-Davis-Gundy and Young inequalities,

$$
\begin{aligned}
\boldsymbol{P}\left\{\left|Z_{k}\right|_{p} \geq \Lambda\right\} & \leq \frac{1}{\Lambda^{p}} \boldsymbol{E} \int_{0}^{T}\left\|Z_{k}(t)\right\|^{p} \mathrm{~d} t \\
& \leq \frac{D_{2}}{\Lambda^{p}} \boldsymbol{E} \int_{0}^{T}\left(\int_{0}^{t}(t-s)^{-2 \alpha}\left\|\sigma_{k}\left(s, X_{k}(s)\right)\right\|^{2} \mathrm{~d} s\right)^{p / 2} \mathrm{~d} t \\
& \leq \frac{D_{2}}{\Lambda^{p}}\left(\int_{0}^{T} s^{-2 \alpha} \mathrm{d} s\right)^{p / 2}\left(\int_{0}^{T} \boldsymbol{E}\left\|\sigma_{k}\left(s, X_{k}(s)\right)\right\|^{p} \mathrm{~d} s\right) \\
& \leq \frac{D_{3}}{\Lambda^{p}}\left(1+\boldsymbol{E}\|\varphi\|^{p}\right) .
\end{aligned}
$$


Let us choose $\Lambda_{0}<\infty$ so that

$$
\frac{D_{1}+D_{3}}{\Lambda_{0}^{p}}\left(1+\boldsymbol{E}\|\varphi\|^{p}\right)<\frac{\varepsilon}{3}
$$

and set

$$
\begin{aligned}
K=\left\{f \in \mathscr{C}\left([0, T] ; \mathbb{R}^{m}\right) ;\right. & f=x+R_{1} r+\frac{\sin \pi \alpha}{\pi} R_{\alpha} v, x \in \Gamma \\
r, v & \left.\in L^{p}\left(0, T ; \mathbb{R}^{m}\right),|r|_{p} \vee|v|_{p} \leq \Lambda_{0}\right\}
\end{aligned}
$$

Since the operators $R_{1}$ and $R_{\alpha}$ are compact, the set $K$ is relatively compact and

$$
\begin{aligned}
\boldsymbol{P}\left\{X_{k} \notin K\right\} & \leq \boldsymbol{P}\{\varphi \notin \Gamma\}+\boldsymbol{P}\left\{\left|b_{k}\left(\cdot, X_{k}(\cdot)\right)\right|_{p}>\Lambda_{0}\right\}+\boldsymbol{P}\left\{\left|Z_{k}\right|_{p}>\Lambda_{0}\right\} \\
& \leq \frac{2}{3} \varepsilon<\varepsilon
\end{aligned}
$$

for any $k \geq 1$, which completes the proof of tightness under the additional assumption (2.3).

Finally, let $\varphi$ be arbitrary. Let $\varepsilon>0$ be fixed, we may find $\Pi \geq 0$ such that $\boldsymbol{P}\{\|\varphi\|>\Pi\}<\frac{\varepsilon}{2}$. Let $\hat{X}_{k}, k \geq 1$, be the solutions to

$$
\mathrm{d} \hat{X}_{k}=b_{k}\left(t, \hat{X}_{k}\right) \mathrm{d} t+\sigma_{k}\left(t, \hat{X}_{k}\right) \mathrm{d} W, \quad \hat{X}_{k}(0)=\mathbf{1}_{\{\|\varphi\| \leq \Pi\}} \varphi .
$$

The initial condition in (2.4) satisfies (2.3), so by the first part of the proof we know that the set $\left\{\boldsymbol{P} \circ \hat{X}_{k}^{-1} ; k \geq 1\right\}$ is tight and there exists a compact set $K \subseteq \mathscr{C}_{m}$ such that

$$
\inf _{k \geq 1} \boldsymbol{P}\left\{\hat{X}_{k} \notin K\right\} \leq \frac{\varepsilon}{2} .
$$

Since the coefficients $b_{k}, \sigma_{k}$ are Lipschitz continuous in space variables,

$$
\mathbf{1}_{\{\|\varphi\| \leq \Pi\}} \hat{X}_{k}=\mathbf{1}_{\{\|\varphi\| \leq \Pi\}} X_{k} \quad \boldsymbol{P} \text {-almost surely }
$$

for all $k \geq 1$, this implies

$$
\boldsymbol{P}\left\{X_{k} \notin K\right\} \leq \boldsymbol{P}\left\{\hat{X}_{k} \notin K\right\}+\boldsymbol{P}\{\|\varphi\|>\Pi\}<\varepsilon
$$

for any $k \geq 1$ and tightness of the set $\left\{\boldsymbol{P} \circ X_{k}^{-1} ; k \geq 1\right\}$ follows. Q.E.D.

Corollary 2.6. The set $\left\{\boldsymbol{P} \circ\left(X_{k}, W\right)^{-1} ; k \geq 1\right\}$ is a tight set of probability measures on $\mathscr{C}\left([0, T] ; \mathbb{R}^{m}\right) \times \mathscr{C}\left([0, T] ; \mathbb{R}^{n}\right)$.

By the Prokhorov theorem, the set $\left\{\boldsymbol{P} \circ\left(X_{k}, W\right)^{-1} ; k \geq 1\right\}$ is relatively (sequentially) compact in the weak topology of probability measures, so it contains 
a weakly convergent subsequence. Without loss of generality we may (and shall) assume that the sequence $\left\{\boldsymbol{P} \circ\left(X_{k}, W\right)^{-1}\right\}_{k=1}^{\infty}$ itself is weakly convergent. Let us set for brevity $\tilde{\boldsymbol{P}}_{k}=\boldsymbol{P} \circ\left(X_{k}, W\right)^{-1}, k \geq 1$, and denote the weak limit of $\left\{\tilde{\boldsymbol{P}}_{k}\right\}_{k=1}^{\infty}$ by $\tilde{\boldsymbol{P}}_{0}$. Set further

$$
U=\mathscr{C}_{m} \times \mathscr{C}_{n}, \quad \mathscr{U}=\operatorname{Borel}\left(\mathscr{C}_{m}\right) \otimes \operatorname{Borel}\left(\mathscr{C}_{n}\right),
$$

and let $(Y, B)$ be the process of projections on $U$, that is

$$
\left(Y_{t}, B_{t}\right): \mathscr{C}_{m} \times \mathscr{C}_{n} \longrightarrow \mathbb{R}^{m} \times \mathbb{R}^{n},(h, g) \longmapsto(h(t), g(t)), \quad 0 \leq t \leq T .
$$

Finally, let $\left(\mathscr{U}_{t}\right)$ be the $\tilde{\boldsymbol{P}}_{0}$-augmented canonical filtration of the process $(Y, B)$, that is

$$
\mathscr{U}_{t}=\sigma\left(\sigma\left(\varrho_{t} Y, \varrho_{t} B\right) \cup\left\{N \in \mathscr{U} ; \tilde{\boldsymbol{P}}_{0}(N)=0\right\}\right), 0 \leq t \leq T .
$$

3. IdEntification of the Limit. In this section we shall show that $((U, \mathscr{U}$, $\left.\left.\left(\mathscr{U}_{t}\right), \tilde{\boldsymbol{P}}_{0}\right), B, Y\right)$ is a weak solution to the problem $(0.7)$. Towards this end, define

$$
M_{k}=Y-Y(0)-\int_{0}^{\cdot} b_{k}(r, Y(r)) \mathrm{d} r, \quad k \geq 0,
$$

where we set $b_{0}=b, \sigma_{0}=\sigma$. The proof is an immediate consequence of the following four lemmas.

Lemma 3.1. The process $M_{0}$ is an m-dimensional local $\left(\mathscr{U}_{t}\right)$-martingale on $\left(U, \mathscr{U}, \tilde{\boldsymbol{P}}_{0}\right)$.

Lemma 3.2. The process $B$ is an $n$-dimensional $\left(\mathscr{U}_{t}\right)$-Wiener process on $\left(U, \mathscr{U}, \tilde{\boldsymbol{P}}_{0}\right)$.

Lemma 3.3. The process

$$
\left\|M_{0}\right\|^{2}-\int_{0}^{\cdot}\|\sigma(r, Y(r))\|^{2} \mathrm{~d} r
$$

is a local $\left(\mathscr{U}_{t}\right)$-martingale on $\left(U, \mathscr{U}, \tilde{\boldsymbol{P}}_{0}\right)$.

Lemma 3.4. The process

$$
M_{0} \otimes B-\int_{0}^{\cdot} \sigma(r, Y(r)) \mathrm{d} r
$$

is an $\mathbb{M}_{m \times n}$-valued local $\left(\mathscr{U}_{t}\right)$-martingale on $\left(U, \mathscr{U}, \tilde{\boldsymbol{P}}_{0}\right)$. 
Proofs of these lemmas have an identical structure, so we prove only the first of them in detail, the other ones being treated only in a concise manner. In the course of the proof, we shall need two easy results on continuity properties of the first entrance times as functionals of paths. Let $V \geq 1$, for any $L \in \mathbb{R}_{+}$define

$$
\tau_{L}: \mathscr{C}_{V} \longrightarrow[0, T], f \longmapsto \inf \{t \geq 0 ;\|f(t)\| \geq L\}
$$

(with a convention $\inf \emptyset=T$ ).

Lemma 3.5. (a) For any $f \in \mathscr{C}_{V}$, the function $L \longmapsto \tau_{L}(f)$ is nondecreasing and left-continuous on $\mathbb{R}_{+}$.

(b) For each $L \in \mathbb{R}_{+}$, the mapping $\tau_{L}$ is lower semicontinuous. Moreover, $\tau_{L}$ is continuous at every point $f \in \mathscr{C}_{V}$ for which $\tau_{\bullet}(f)$ is continuous at $L$.

If $\left(Z_{t}\right)_{t \in[0, T]}$ is a continuous $\mathbb{R}^{V}$-valued stochastic process defined on a probability space $(G, \mathscr{G}, \boldsymbol{q})$, then $\left(\tau_{L}(Z)\right)_{L \geq 0}$ is a stochastic process with nondecreasing left-continuous trajectories, whence we get

Lemma 3.6. The set

$$
\left\{L \in \mathbb{R}_{+} ; \boldsymbol{q}\left\{\tau_{\bullet}(Z) \text { is not continuous at } L\right\}>0\right\}
$$

is at most countable.

Lemma 3.5 is proved (but not stated exactly in this form) in [11], see Lemma VI.2.10 and Proposition VI.2.11 there. For Lemma 3.6, see [11], Lemma VI.3.12. In the book [11], $\tau_{L}$ is considered as a function on the Skorokhod space $\mathbb{D}$, in our case the proofs simplify further; they are recalled in Appendix to keep the paper self-contained.

Further, let us quote an useful result on weak convergence of measures (cf. e.g. [2], Proposition IX.5.7).

Lemma 3.7. Let $\left\{\nu_{r}\right\}_{r \geq 1}$ be a sequence of Borel probability measures on a metric space $\Theta$ converging weakly to a Borel probability measure $\nu_{0}$. Let $f: \Theta \longrightarrow \mathbb{R}$ be a bounded real function continuous at $\nu_{0}$-almost all points of $\Theta$. Then

$$
\lim _{r \rightarrow \infty} \int_{\Theta} f \mathrm{~d} \nu_{r}=\int_{\Theta} f \mathrm{~d} \nu_{0}
$$

Proof of Lemma 3.1. The idea of the proof is simple: define processes

$$
\mu_{k}=X_{k}-X_{k}(0)-\int_{0}^{\cdot} b_{k}\left(r, X_{k}(r)\right) \mathrm{d} r, \quad k \geq 1
$$

in analogy with the definition of $M_{k}$ but using the solutions $X_{k}$ to the problem (1.2) instead of the process $Y$. We shall prove: i) $\mu_{k}, k \geq 1$, are local martingales, 
ii) $M_{k}, k \geq 1$, are local martingales with respect to the measure $\tilde{\boldsymbol{P}}_{k}$ due to the equality of laws $\tilde{\boldsymbol{P}}_{k} \circ(Y, B)^{-1}=\boldsymbol{P} \circ\left(X_{k}, W\right)^{-1}$, iii) $M_{0}$ is a local martingale as a limit of local martingales $M_{k}$.

First, as $X_{k}$ solves (1.2),

$$
\mu_{k}(t)=\int_{0}^{t} \sigma_{k}\left(r, X_{k}(r)\right) \mathrm{d} W_{r}, \quad 0 \leq t \leq T
$$

and so $\mu_{k}$ is a local $\left(\mathscr{F}_{t}\right)$-martingale. Take an $L \in \mathbb{R}_{+}$, for the time being arbitrary. Obviously, $\tau_{L}\left(X_{k}\right)$ is a stopping time and $\mu_{k}\left(\cdot \wedge \tau_{L}\left(X_{k}\right)\right)$ is a bounded process by (1.1) and the definition of $\tau_{L}$, hence $\mu_{k}\left(\cdot \wedge \tau_{L}\left(X_{k}\right)\right)$ is a martingale.

Hereafter, times $s, t \in[0, T], s \leq t$, and a continuous function

$$
\gamma: \mathscr{C}\left([0, s] ; \mathbb{R}^{m}\right) \times \mathscr{C}\left([0, s] ; \mathbb{R}^{n}\right) \longrightarrow[0,1]
$$

will be fixed but otherwise arbitrary. Obviously, $\gamma\left(\varrho_{s} X_{k}, \varrho_{s} W\right)$ is a bounded $\mathscr{F}_{s^{-}}$ measurable function, hence

$$
\boldsymbol{E} \gamma\left(\varrho_{s} X_{k}, \varrho_{s} W\right) \mu_{k}\left(t \wedge \tau_{L}\left(X_{k}\right)\right)=\boldsymbol{E} \gamma\left(\varrho_{s} X_{k}, \varrho_{s} W\right) \mu_{k}\left(s \wedge \tau_{L}\left(X_{k}\right)\right)
$$

by the martingale property of $\mu_{k}\left(\cdot \wedge \tau_{L}\left(X_{k}\right)\right)$.

Note that the mapping

$$
[0, T] \times \mathscr{C}_{m} \longrightarrow \mathbb{R}^{m},(u, h) \longmapsto h(u)-h(0)-\int_{0}^{u} b_{k}(r, h(r)) \mathrm{d} r
$$

is continuous for any $k \geq 0$ due to the continuity of $b_{k}(r, \cdot)$, and the mapping

$$
\mathscr{C}_{m} \longrightarrow[0, T] \times \mathscr{C}_{m}, h \longmapsto\left(\xi \wedge \tau_{L}(h), h\right)
$$

is Borel for any $\xi \in[0, T]$ fixed by Lemma 3.5(b), thus also their superposition

$$
H_{k}(\xi, \cdot): \mathscr{C}_{m} \longrightarrow \mathbb{R}^{m}, h \longmapsto h\left(\xi \wedge \tau_{L}(h)\right)-h(0)-\int_{0}^{\xi \wedge \tau_{L}(h)} b_{k}(r, h(r)) \mathrm{d} r
$$

is Borel. Consequently, the mapping

$$
\mathscr{C}_{m} \times \mathscr{C}_{n} \longrightarrow \mathbb{R}^{m},(h, g) \longmapsto \gamma\left(\varrho_{s} h, \varrho_{s} g\right) H_{k}(\xi, h)
$$

is Borel. Since $\mu_{k}\left(\xi \wedge \tau_{L}\left(X_{k}\right)\right)=H_{k}\left(\xi, X_{k}\right), k \geq 1$, and $M_{k}\left(\xi \wedge \tau_{L}(Y)\right)=H_{k}(\xi, Y)$, $k \geq 0$, we get

$$
\boldsymbol{P} \circ\left[\gamma\left(\varrho_{s} X_{k}, \varrho_{s} W\right) \mu_{k}\left(\xi \wedge \tau_{L}\left(X_{k}\right)\right)\right]^{-1}=\tilde{\boldsymbol{P}}_{k} \circ\left[\gamma\left(\varrho_{s} Y, \varrho_{s} B\right) M_{k}\left(\xi \wedge \tau_{L}(Y)\right)\right]^{-1}
$$


for all $k \geq 1$ by the definition of $\tilde{\boldsymbol{P}}_{k}$, which together with (3.1) implies

$$
\tilde{\boldsymbol{E}}_{k} \gamma\left(\varrho_{s} Y, \varrho_{s} B\right) M_{k}\left(t \wedge \tau_{L}(Y)\right)=\tilde{\boldsymbol{E}}_{k} \gamma\left(\varrho_{s} Y, \varrho_{s} B\right) M_{k}\left(s \wedge \tau_{L}(Y)\right), k \geq 1 .
$$

Now, suppose in addition that $L$ is chosen so that

$$
\tilde{\boldsymbol{P}}_{0}\left\{\tau_{\bullet}(Y) \text { is continuous at } L\right\}=1 \text {. }
$$

(Lemma 3.6 shows that such a choice is possible.) Then

$$
\tilde{\boldsymbol{P}}_{0}\left\{(f, g) \in U ; \tau_{L}(\cdot) \text { is continuous at } f\right\}=1
$$

by Lemma $3.5(\mathrm{~b})$ and the fact that $Y$ is a canonical projection from $U$ onto $\mathscr{C}_{m}$, so also

$$
\tilde{\boldsymbol{P}}_{0}\left\{(f, g) \in U ; H_{0}(\xi, \cdot) \text { is continuous at } f\right\}=1 .
$$

This implies that $\gamma\left(\varrho_{s} Y, \varrho_{s} B\right) H_{0}(\xi, Y)$ is a bounded function continuous $\tilde{\boldsymbol{P}}_{0}$-almost everywhere on $U$ for any $\xi$ fixed. We may estimate

$$
\begin{aligned}
\| \tilde{\boldsymbol{E}}_{k} \gamma\left(\varrho_{s} Y, \varrho_{s} B\right) H_{k}(\xi, & Y)-\tilde{\boldsymbol{E}}_{0} \gamma\left(\varrho_{s} Y, \varrho_{s} B\right) H_{0}(\xi, Y) \| \\
\leq & \left\|\tilde{\boldsymbol{E}}_{k} \gamma\left(\varrho_{s} Y, \varrho_{s} B\right)\left[H_{k}(\xi, Y)-H_{0}(\xi, Y)\right]\right\| \\
& +\left\|\tilde{\boldsymbol{E}}_{k} \gamma\left(\varrho_{s} Y, \varrho_{s} B\right) H_{0}(\xi, Y)-\tilde{\boldsymbol{E}}_{0} \gamma\left(\varrho_{s} Y, \varrho_{s} B\right) H_{0}(\xi, Y)\right\| .
\end{aligned}
$$

From Lemma 3.7 we obtain that

$$
\lim _{k \rightarrow \infty} \tilde{\boldsymbol{E}}_{k} \gamma\left(\varrho_{s} Y, \varrho_{s} B\right) H_{0}(\xi, Y)=\tilde{\boldsymbol{E}}_{0} \gamma\left(\varrho_{s} Y, \varrho_{s} B\right) H_{0}(\xi, Y)
$$

Further,

$$
\begin{aligned}
&\left\|\tilde{\boldsymbol{E}}_{k} \gamma\left(\varrho_{s} Y, \varrho_{s} B\right)\left[H_{k}(\xi, Y)-H_{0}(\xi, Y)\right]\right\| \\
& \leq \tilde{\boldsymbol{E}}_{k}\left\|H_{k}(\xi, Y)-H_{0}(\xi, Y)\right\| \\
&=\tilde{\boldsymbol{E}}_{k}\left\|\int_{0}^{\xi \wedge \tau_{L}(Y)}\left[b_{k}(r, Y(r))-b_{0}(r, Y(r))\right] \mathrm{d} r\right\| \\
&=\tilde{\boldsymbol{E}}_{k} \mathbf{1}_{\left\{\tau_{L}(Y)>0\right\}}\left\|\int_{0}^{\xi \wedge \tau_{L}(Y)}\left[b_{k}(r, Y(r))-b_{0}(r, Y(r))\right] \mathrm{d} r\right\| \\
& \leq \tilde{\boldsymbol{E}}_{k} \mathbf{1}_{\left\{\tau_{L}(Y)>0\right\}} \int_{0}^{\xi \wedge \tau_{L}(Y)}\left\|b_{k}(r, Y(r))-b_{0}(r, Y(r))\right\| \mathrm{d} r \\
& \leq \tilde{\boldsymbol{E}}_{k} \mathbf{1}_{\left\{\tau_{L}(Y)>0\right\}} \int_{0}^{T}\left\|b_{k}\left(r, Y\left(r \wedge \tau_{L}(Y)\right)\right)-b_{0}\left(r, Y\left(r \wedge \tau_{L}(Y)\right)\right)\right\| \mathrm{d} r \\
& \leq \tilde{\boldsymbol{E}}_{k} \mathbf{1}_{\left\{\tau_{L}(Y)>0\right\}} \int_{0}^{T} \sup _{\|z\| \leq L}\left\|b_{k}(r, z)-b_{0}(r, z)\right\| \mathrm{d} r \\
& \leq \int_{0}^{T} \sup _{\|z\| \leq L}\left\|b_{k}(r, z)-b_{0}(r, z)\right\| \mathrm{d} r,
\end{aligned}
$$


as $\left\|Y\left(r \wedge \tau_{L}(Y)\right)\right\| \leq L$ on the set $\left\{\tau_{L}(Y)>0\right\}$. Since $b_{k}(r, \cdot) \rightarrow b_{0}(r, \cdot)$ locally uniformly on $\mathbb{R}^{m}$ for every $r \in[0, T]$ and

$$
\sup _{\|z\| \leq L}\left\|b_{k}(r, z)-b_{0}(r, z)\right\| \leq 2 K_{*}(2+L)
$$

by (0.6) and (1.1), we have

$$
\lim _{k \rightarrow \infty} \int_{0}^{T} \sup _{\|z\| \leq L}\left\|b_{k}(r, z)-b_{0}(r, z)\right\| \mathrm{d} r=0
$$

by the dominated convergence theorem, hence

$$
\lim _{k \rightarrow \infty} \tilde{\boldsymbol{E}}_{k} \gamma\left(\varrho_{s} Y, \varrho_{s} B\right) H_{k}(\xi, Y)=\tilde{\boldsymbol{E}}_{0} \gamma\left(\varrho_{s} Y, \varrho_{s} B\right) H_{0}(\xi, Y)
$$

for any $\xi \in[0, T]$. Therefore,

$$
\tilde{\boldsymbol{E}}_{0} \gamma\left(\varrho_{s} Y, \varrho_{s} B\right) M_{0}\left(t \wedge \tau_{L}(Y)\right)=\tilde{\boldsymbol{E}}_{0} \gamma\left(\varrho_{s} Y, \varrho_{s} B\right) M_{0}\left(s \wedge \tau_{L}(Y)\right)
$$

follows from (3.2). If $G \subseteq \mathscr{C}\left([0, s] ; \mathbb{R}^{m} \times \mathbb{R}^{n}\right)$ is an arbitrary open set, then there exist continuous functions $g_{l}: \mathscr{C}\left([0, s] ; \mathbb{R}^{m} \times \mathbb{R}^{n}\right) \longrightarrow[0,1]$ such that $g_{l} \nearrow \mathbf{1}_{G}$ on $\mathscr{C}\left([0, s] ; \mathbb{R}^{m} \times \mathbb{R}^{n}\right)$ as $l \rightarrow \infty$. Therefore, using the Levi monotone convergence theorem we derive from (3.4) that

$$
\tilde{\boldsymbol{E}}_{0} \mathbf{1}_{G}\left(\varrho_{s} Y, \varrho_{s} B\right) M_{0}\left(t \wedge \tau_{L}(Y)\right)=\tilde{\boldsymbol{E}}_{0} \mathbf{1}_{G}\left(\varrho_{s} Y, \varrho_{s} B\right) M_{0}\left(s \wedge \tau_{L}(Y)\right)
$$

Further,

$$
\left\{G \subseteq \mathscr{C}\left([0, s] ; \mathbb{R}^{m} \times \mathbb{R}^{n}\right) ; \quad G \text { Borel and (3.5) holds for } \mathbf{1}_{G}\right\}
$$

is a $\lambda$-system containing, as we have just shown, the system of all open sets in $\mathscr{C}\left([0, s] ; \mathbb{R}^{m} \times \mathbb{R}^{n}\right)$ closed under finite intersections. Consequently, (3.5) holds for all Borel sets $G \subseteq \mathscr{C}\left([0, s] ; \mathbb{R}^{m} \times \mathbb{R}^{n}\right)$, that is

$$
\tilde{\boldsymbol{E}}_{0} \mathbf{1}_{A} M_{0}\left(t \wedge \tau_{L}(Y)\right)=\tilde{\boldsymbol{E}}_{0} \mathbf{1}_{A} M_{0}\left(s \wedge \tau_{L}(Y)\right)
$$

holds for all $A \in \sigma\left(\varrho_{s} Y, \varrho_{s} B\right)$, thus for all $A \in \mathscr{U}_{s}$. We see that $M_{0}\left(\cdot \wedge \tau_{L}(Y)\right)$ is a $\left(\mathscr{U}_{t}\right)$-martingale, whenever $L \in \mathbb{R}_{+}$satisfies (3.3). It remains to note that by Lemma 3.6 there exists a sequence $L_{r} \nearrow \infty$ such that

$$
\tilde{\boldsymbol{P}}_{0}\left\{\tau_{\bullet}(Y) \text { is continuous at } L_{r} \text { for every } r \geq 1\right\}=1 \text {. }
$$


As $\left\{\tau_{L_{r}}(Y)\right\}$ is plainly a localizing sequence of stopping times, we conclude that $M_{0}$ is a local $\left(\mathscr{U}_{t}\right)$-martingale on $\left(U, \mathscr{U}, \tilde{\boldsymbol{P}}_{0}\right)$, as claimed. Q.E.D.

Proof of Lemma 3.2. By our construction, $\boldsymbol{P} \circ W^{-1}=\tilde{\boldsymbol{P}}_{k} \circ B^{-1}$ for each $k \geq 1$, so also $\boldsymbol{P} \circ W^{-1}=\tilde{\boldsymbol{P}}_{0} \circ B^{-1}$ and $B$ is an $n$-dimensional Wiener process (with respect to its canonical filtration) on $\left(U, \mathscr{U}, \tilde{\boldsymbol{P}}_{0}\right)$. In particular, its tensor quadratic variation satisfies $\langle\langle B\rangle\rangle_{t}=t I$. Mimicking the procedure from the previous proof we may check easily that $B$ is a local $\left(\mathscr{U}_{t}\right)$-martingale, hence an $\left(\mathscr{U}_{t}\right)$-Wiener process by the Lévy theorem. Q.E.D.

Proof of Lemma 3.3. We know that $\mu_{k}, k \geq 1$, are local martingales and

$$
\left\langle\mu_{k}\right\rangle=\left\langle\int_{0}^{\cdot} \sigma_{k}\left(r, X_{k}(r)\right) \mathrm{d} W_{r}\right\rangle=\int_{0}^{\cdot}\left\|\sigma_{k}\left(r, X_{k}(r)\right)\right\|^{2} \mathrm{~d} r
$$

thus

$$
\left\|\mu_{k}\right\|^{2}-\int_{0}^{\cdot}\left\|\sigma_{k}\left(r, X_{k}(r)\right)\right\|^{2} \mathrm{~d} r, \quad k \geq 1
$$

are continuous local martingales. For times $s \leq t$ and a function $\gamma$ introduced in the proof of Lemma 3.1 we get

$$
\begin{aligned}
& \boldsymbol{E} \gamma\left(\varrho_{s} X_{k}, \varrho_{s} W\right)\left[\left\|\mu_{k}\left(t \wedge \tau_{L}\left(X_{k}\right)\right)\right\|^{2}-\int_{0}^{t \wedge \tau_{L}\left(X_{k}\right)}\left\|\sigma_{k}\left(r, X_{k}(r)\right)\right\|^{2} \mathrm{~d} r\right] \\
& =\boldsymbol{E} \gamma\left(\varrho_{s} X_{k}, \varrho_{s} W\right)\left[\left\|\mu_{k}\left(s \wedge \tau_{L}\left(X_{k}\right)\right)\right\|^{2}-\int_{0}^{s \wedge \tau_{L}\left(X_{k}\right)}\left\|\sigma_{k}\left(r, X_{k}(r)\right)\right\|^{2} \mathrm{~d} r\right] .
\end{aligned}
$$

Note that

$$
\mathscr{C}_{m} \longrightarrow \mathbb{R}, h \longmapsto\left\|H_{k}(\xi, h)\right\|^{2}-\int_{0}^{\xi \wedge \tau_{L}(h)}\left\|\sigma_{k}(r, h(r))\right\|^{2} \mathrm{~d} r
$$

is a Borel mapping for all $k \geq 0$ and $\xi \in[0, T]$. It can be seen easily that it suffices to check that

$$
\mathscr{C}_{m} \longrightarrow \mathbb{R}, h \longmapsto \int_{0}^{u}\left\|\sigma_{k}(r, h(r))\right\|^{2} \mathrm{~d} r
$$

is a continuous mapping for any $u \in[0, T]$; this follows from the estimate

$$
\begin{aligned}
& \left|\int_{0}^{u}\left\|\sigma_{k}\left(r, h_{1}(r)\right)\right\|^{2} \mathrm{~d} r-\int_{0}^{u}\left\|\sigma_{k}\left(r, h_{2}(r)\right)\right\|^{2} \mathrm{~d} r\right| \\
& \quad \leq \int_{0}^{u}\left\{\left\|\sigma_{k}\left(r, h_{1}(r)\right)\right\|+\left\|\sigma_{k}\left(r, h_{2}(r)\right)\right\|\right\}\left|\left\|\sigma_{k}\left(r, h_{1}(r)\right)\right\|-\left\|\sigma_{k}\left(r, h_{2}(r)\right)\right\|\right| \mathrm{d} r \\
& \quad \leq K_{*}\left(4+\left\|h_{1}\right\|_{\mathscr{C}_{m}}+\left\|h_{2}\right\| \mathscr{C}_{m}\right) \int_{0}^{u}\left\|\sigma_{k}\left(r, h_{1}(r)\right)-\sigma_{k}\left(r, h_{2}(r)\right)\right\| \mathrm{d} r
\end{aligned}
$$


for $h_{1}, h_{2} \in \mathscr{C}_{m}$, continuity of functions $\sigma_{k}(r, \cdot)$ and the dominated convergence theorem.

Hence (3.6) yields

$$
\begin{aligned}
\tilde{\boldsymbol{E}}_{k} \gamma\left(\varrho_{s} Y, \varrho_{s} B\right)\left[\left\|M_{k}\left(t \wedge \tau_{L}(Y)\right)\right\|^{2}-\int_{0}^{t \wedge \tau_{L}(Y)}\left\|\sigma_{k}(r, Y(r))\right\|^{2} \mathrm{~d} r\right] \\
=\tilde{\boldsymbol{E}}_{k} \gamma\left(\varrho_{s} Y, \varrho_{s} B\right)\left[\left\|M_{k}\left(s \wedge \tau_{L}(Y)\right)\right\|^{2}-\int_{0}^{s \wedge \tau_{L}(Y)}\left\|\sigma_{k}(r, Y(r))\right\|^{2} \mathrm{~d} r\right] .
\end{aligned}
$$

Passing to the limit exactly in the same way as in the proof of Lemma 3.1 we obtain

$$
\begin{aligned}
\tilde{\boldsymbol{E}}_{0} \gamma\left(\varrho_{s} Y, \varrho_{s} B\right)\left[\left\|M_{0}\left(t \wedge \tau_{L}(Y)\right)\right\|^{2}-\int_{0}^{t \wedge \tau_{L}(Y)}\left\|\sigma_{0}(r, Y(r))\right\|^{2} \mathrm{~d} r\right] \\
=\tilde{\boldsymbol{E}}_{0} \gamma\left(\varrho_{s} Y, \varrho_{s} B\right)\left[\left\|M_{0}\left(s \wedge \tau_{L}(Y)\right)\right\|^{2}-\int_{0}^{s \wedge \tau_{L}(Y)}\left\|\sigma_{0}(r, Y(r))\right\|^{2} \mathrm{~d} r\right]
\end{aligned}
$$

provided that $L \in \mathbb{R}_{+}$satisfies (3.3), and the proof may be completed easily. Q.E.D.

Proof of Lemma 3.4. Since $\mu_{k}$ and $W$ are continuous local martingales, the process $\mu_{k} \otimes W-\left\langle\left\langle\mu_{k}, W\right\rangle\right.$ is an $\mathbb{M}_{m \times n}$-valued local martingale. Let us denote $\mu_{k}=\left(\mu_{k}^{i}\right)_{i=1}^{m}, W=\left(W^{j}\right)_{j=1}^{n}$ and $\sigma_{k}=\left(\sigma_{k}^{i j}\right)_{i=1}^{m} \underset{j=1}{n}$. Then

$$
\begin{aligned}
\left\langle\mu_{k}^{i}, W^{j}\right\rangle & =\left\langle\sum_{l=1}^{n} \int_{0}^{\cdot} \sigma_{k}^{i l}\left(r, X_{k}(r)\right) \mathrm{d} W^{l}(r), W^{j}\right\rangle \\
& =\sum_{l=1}^{n} \int_{0}^{\cdot} \sigma_{k}^{i l}\left(r, X_{k}(r)\right) \mathrm{d}\left\langle W^{l}, W^{j}\right\rangle_{r} \\
& =\int_{0}^{\cdot} \sigma_{k}^{i j}\left(r, X_{k}(r)\right) \mathrm{d} r
\end{aligned}
$$

therefore,

$$
\mu_{k} \otimes W-\int_{0}^{\cdot} \sigma_{k}\left(r, X_{k}(r)\right) \mathrm{d} r
$$

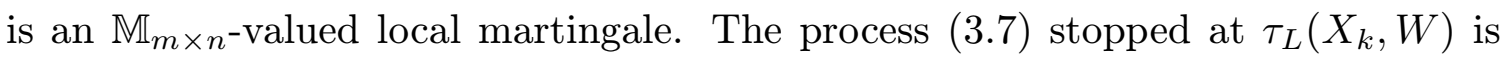
bounded, hence it is a martingale and so

$$
\begin{aligned}
& \boldsymbol{E} \gamma\left(\varrho_{s} X_{k}, \varrho_{s} W\right)\left[\left(\mu_{k} \otimes W\right)\left(t \wedge \tau_{L}\left(X_{k}, W\right)\right)-\int_{0}^{t \wedge \tau_{L}\left(X_{k}, W\right)} \sigma_{k}\left(r, X_{k}(r)\right) \mathrm{d} r\right] \\
& =\boldsymbol{E} \gamma\left(\varrho_{s} X_{k}, \varrho_{s} W\right)\left[\left(\mu_{k} \otimes W\right)\left(s \wedge \tau_{L}\left(X_{k}, W\right)\right)-\int_{0}^{s \wedge \tau_{L}\left(X_{k}, W\right)} \sigma_{k}\left(r, X_{k}(r)\right) \mathrm{d} r\right],
\end{aligned}
$$


whenever $0 \leq s \leq t \leq T$ and $\gamma$ is a continuous function as above. (Since $\mathscr{C}_{m} \times \mathscr{C}_{n} \cong$ $\mathscr{C}_{m+n}$, it is clear how $\tau_{L}(f, g)$ is defined for $(f, g) \in \mathscr{C}_{m} \times \mathscr{C}_{n}$.) Now we may proceed as in the proof of Lemma 3.1. Q.E.D.

Proof of Theorem 0.1. Lemmas 3.1-3.4 having been established, it is straightforward to prove that $\left(\left(U, \mathscr{U},\left(\mathscr{U}_{t}\right), \tilde{\boldsymbol{P}}_{0}\right), B, Y\right)$ is a weak solution of (0.7). Since $\tilde{\boldsymbol{P}}_{0} \circ Y(0)^{-1}=\tilde{\boldsymbol{P}}_{k} \circ Y(0)^{-1}=\boldsymbol{P} \circ \varphi^{-1}=\nu$ by our construction, it remains only to show that

$$
Y(t)=Y(0)+\int_{0}^{t} b(r, Y(r)) \mathrm{d} r+\int_{0}^{t} \sigma(r, Y(r)) \mathrm{d} B(r)
$$

for any $t \in[0, T] \tilde{\boldsymbol{P}}_{0}$-almost surely, that is

$$
M_{0}(t)=\int_{0}^{t} \sigma(r, Y(r)) \mathrm{d} B(r) \quad \text { for all } t \in[0, T] \tilde{\boldsymbol{P}}_{0} \text {-almost surely. }
$$

Obviously, (3.8) is equivalent to

$$
\left\langle M_{0}-\int_{0}^{\cdot} \sigma(r, Y(r)) \mathrm{d} B(r)\right\rangle_{T}=0 \quad \tilde{\boldsymbol{P}}_{0} \text {-almost surely. }
$$

We have

$$
\begin{aligned}
\left\langle M_{0}-\int_{0}^{\sigma} \sigma(r, Y(r)) \mathrm{d} B(r)\right\rangle_{T}= & \left\langle M_{0}\right\rangle_{T}+\left\langle\int_{0}^{\cdot} \sigma(r, Y(r)) \mathrm{d} B(r)\right\rangle_{T} \\
& -2 \sum_{i=1}^{m}\left\langle M_{0}^{i}, \sum_{j=1}^{n} \int_{0}^{\cdot} \sigma^{i j}(r, Y(r)) \mathrm{d} B^{j}(r)\right\rangle_{T} \\
= & \left\langle M_{0}\right\rangle_{T}+\int_{0}^{T}\|\sigma(r, Y(r))\|^{2} \mathrm{~d} r \\
& -2 \sum_{i=1}^{m}\left\langle M_{0}^{i}, \sum_{j=1}^{n} \int_{0}^{.} \sigma^{i j}(r, Y(r)) \mathrm{d} B^{j}(r)\right\rangle_{T} .
\end{aligned}
$$

By Lemma 3.3,

$$
\left\langle M_{0}\right\rangle_{T}=\int_{0}^{T}\|\sigma(r, X(r))\|^{2} \mathrm{~d} r
$$

and by Lemma 3.4 we obtain

$$
\begin{aligned}
\sum_{i=1}^{m} \sum_{j=1}^{n}\left\langle M_{0}^{i}, \int_{0}^{.} \sigma^{i j}(r, Y(r)) \mathrm{d} B^{j}(r)\right\rangle_{T} & =\sum_{i=1}^{m} \sum_{j=1}^{n} \int_{0}^{T} \sigma^{i j}(r, Y(r)) \mathrm{d}\left\langle M_{0}^{i}, B^{j}\right\rangle_{r} \\
& =\sum_{i=1}^{m} \sum_{j=1}^{n} \int_{0}^{T}\left(\sigma^{i j}(r, Y(r))\right)^{2} \mathrm{~d} r \\
& =\int_{0}^{T}\|\sigma(r, Y(r))\|^{2} \mathrm{~d} r,
\end{aligned}
$$


hence (3.9) holds true. Q.E.D.

Remark 3.1. If the coefficients $b$ and $\sigma$ of the equation (0.7) are defined on $\mathbb{R}_{+} \times \mathbb{R}^{m}$ and satisfy the assumptions of Theorem 0.1 there, then there exists a weak solution to $(0.7)$ defined for all times $t \geq 0$. The proof remains almost the same, only its part concerning tightness requires small modifications. However, it suffices to realize that the space $\mathscr{C}\left(\mathbb{R}_{+} ; \mathbb{R}^{V}\right)$ equipped with the topology of locally uniform convergence is a Polish space whose Borel $\sigma$-algebra is generated by the projections $f \mapsto f(t), t \geq 0$ and whose closed subset $K$ is compact if and only if $\left\{\varrho_{T} f ; f \in K\right\}$ is a compact subset of $\mathscr{C}\left([0, T] ; \mathbb{R}^{V}\right)$ for all $T \geq 0$.

Remark 3.2. Tracing the proofs in Section 3, we can check easily that, unlike the proof of tightness in Section 2, they depend only on the following properties of the coefficients $b=b_{0}, \sigma=\sigma_{0}$ and their approximations $b_{k}, \sigma_{k}$ :

$1^{\circ}$ the functions $b_{k}(r, \cdot), \sigma_{k}(r, \cdot)$ are continuous on $\mathbb{R}^{m}$ for any $r \in[0, T]$ and $k \geq 0$,

$2^{\circ} b_{k}(r, \cdot) \rightarrow b(r, \cdot), \sigma_{k}(r, \cdot) \rightarrow \sigma(r, \cdot)$ locally uniformly on $\mathbb{R}^{m}$ as $k \rightarrow \infty$ for any $r \in[0, T]$,

$3^{\circ}$ the functions $b_{k}, \sigma_{k}$ are locally bounded uniformly in $k \geq 0$, i.e.

$$
\sup _{k \geq 0} \sup _{r \in[0, T]} \sup _{\|z\| \leq L}\left\{\left\|b_{k}(r, z)\right\| \vee\left\|\sigma_{k}(r, z)\right\|\right\}<\infty
$$

for each $L \geq 0$.

As a consequence, Theorem 0.1 remains valid if existence of a suitable Lyapunov function is supposed instead of the linear growth hypothesis. One proceeds as in the proof of Theorem 0.1, approximating the coefficients $b$ and $\sigma$ by bounded continuous functions that satisfy the same Lyapunov estimate as $b$ and $\sigma$. However, the proof of tightness is more technical, although no fundamentally new ideas are needed; details may be found in a companion paper [7].

4. Appendix. To keep the paper self-contained as much as possible, we provide here proofs of Lemmas 3.5 and 3.6.

Proof of Lemma 3.5. Choose $f \in \mathscr{C}_{V}$ and $L>0$ arbitrarily. The function $K \mapsto \tau_{K}(f)$ is obviously nondecreasing, hence it has a left-hand limit at the point $L$ and

$$
\lim _{K \rightarrow L-} \tau_{K}(f) \leq \tau_{L}(f) .
$$

If $\|f\|_{\mathscr{C}_{V}}<L$ then $\|f\|_{\mathscr{C}_{V}}<L-\delta$ for some $\delta>0$ and thus $\tau_{L}(f)=T=\tau_{K}(f)$ for all $K \in[L-\delta, L]$, so we may assume that $\|f\|_{\mathscr{C}_{V}} \geq L$. Then $\left\|f\left(\tau_{K}(f)\right)\right\| \geq K$ for all $K \in[0, L]$ and continuity of $f$ yields

$$
\left\|f\left(\lim _{K \rightarrow L-} \tau_{K}(f)\right)\right\|=\lim _{K \rightarrow L-}\left\|f\left(\tau_{K}(f)\right)\right\| \geq \lim _{K \rightarrow L-} K=L
$$


whence

$$
\tau_{L}(f) \leq \lim _{K \rightarrow L-} \tau_{K}(f),
$$

which together with (4.1) proves the statement (a).

To prove (b), take an arbitrary sequence $\left\{f_{r}\right\}$ in $\mathscr{C}_{V}$ such that $f_{r} \rightarrow f$ uniformly on $[0, T]$ as $r \rightarrow \infty$. Let $\varepsilon>0$, then

$$
\max _{\left[0, \tau_{L}(f)-\varepsilon\right]}\|f\|<L
$$

so there exists $r_{0} \in \mathbb{N}$ such that

$$
\max _{\left[0, \tau_{L}(f)-\varepsilon\right]}\left\|f_{r}\right\|<L
$$

for all $r \geq r_{0}$, thus $\tau_{L}\left(f_{r}\right) \geq \tau_{L}(f)-\varepsilon$ for all $r \geq r_{0}$. Since $\varepsilon$ was arbitrary,

$$
\liminf _{r \rightarrow \infty} \tau_{L}\left(f_{r}\right) \geq \tau_{L}(f)
$$

that is, $\tau_{L}$ is lower semicontinuous at the point $f$.

Finally, assume in addition that $\tau_{\bullet}(f)$ is continuous at the point $L$. If $\tau_{L}(f)=T$ then

$$
T=\tau_{L}(f) \leq \liminf _{r \rightarrow \infty} \tau_{L}\left(f_{r}\right) \leq \limsup _{r \rightarrow \infty} \tau_{L}\left(f_{r}\right) \leq T
$$

(note that $\tau_{L}$ is $[0, T]$-valued) and we are done. So assume that $\tau_{L}(f)<T$ and take an arbitrary $\varepsilon>0$ satisfying $\tau_{L}(f)+\varepsilon<T$. By continuity, a $K>L$ may be found such that $\tau_{K}(f)<\tau_{L}(f)+\varepsilon$. Consequently,

$$
\max _{\left[0, \tau_{L}(f)+\varepsilon\right]}\|f\| \geq K>L
$$

thus

$$
\max _{\left[0, \tau_{L}(f)+\varepsilon\right]}\left\|f_{r}\right\| \geq L
$$

for all $r$ sufficiently large, that is $\tau_{L}\left(f_{r}\right) \leq \tau_{L}(f)+\varepsilon$ for all $r$ sufficiently large, which implies

$$
\limsup _{r \rightarrow \infty} \tau_{L}\left(f_{r}\right) \leq \tau_{L}(f)
$$

and $\tau_{L}$ is upper semicontinuous at $f$. Q.E.D.

Proof of Lemma 3.6. Here we follow the book [11] closely. First, note that for any given $u>0 \boldsymbol{q}$-almost any trajectory of $\tau_{\bullet}(Z)$ has only finitely many jumps of size greater than $u$. For brevity, set

$$
\Delta \tau_{L}(Z)=\lim _{M \rightarrow L+} \tau_{M}(Z)-\tau_{L}(Z)
$$


and define recursively random times

$$
\Sigma_{0}(u)=0, \Sigma_{p}(u)=\inf \left\{L>\Sigma_{p}(u) ; \Delta \tau_{L}(Z)>u\right\}, \quad u>0, p \in \mathbb{N} .
$$

Plainly, the set

$$
\left\{L \geq 0 ; \boldsymbol{q}\left\{\Sigma_{p}(u)=L\right\}>0\right\}
$$

is at most countable for any $p \in \mathbb{N}$ and $u>0$, hence it only remains to note that

$$
\left\{L \geq 0 ; \boldsymbol{q}\left\{\Delta \tau_{L}(Z)>0\right\}>0\right\}=\bigcup_{p=0}^{\infty} \bigcup_{r=1}^{\infty}\left\{L \geq 0 ; \boldsymbol{q}\left\{\Sigma_{p}\left(r^{-1}\right)=L\right\}>0\right\}
$$

Q.E.D.

\section{REFERENCES}

[1] M. T. Barlow: One dimensional stochastic differential equation with no strong solution, $J$. London Math. Soc. (2) 26(1982), 335-347

[2] N. Bourbaki: Integration II, Springer, Berlin 2004

[3] Z. Brzeźniak, M. Ondreját: Strong solutions to stochastic wave equations with values in Riemannian manifolds, J. Funct. Anal. 253(2007), 449-481

[4] G. Da Prato, J. Zabczyk: Stochastic equations in infinite dimensions, Cambridge University Press, Cambridge 1992

[5] R. M. Dudley: Real analysis and probability, Cambridge University Press, Cambridge 2002

[6] D. Gątarek, B. Gołdys: On weak solutions of stochastic equations in Hilbert spaces, Stochastics Stochastics Rep. 46(1994), 41-51

[7] M. Hofmanová, J. Seidler: On weak solutions of stochastic differential equations II. Dissipative drifts, to appear

[8] N. Ikeda, S. Watanabe: Stochastic differential equations and diffusion processes, 2nd ed., North-Holland, Amsterdam 1989

[9] K. Itô: On a stochastic integral equation, Proc. Japan Acad. 22(1946), 32-35

[10] K. Ito: On stochastic differential equations, Mem. Amer. Math. Soc. 4(1951), 1-51

[11] J. Jacod, A. N. Shiryaev: Limit theorems for stochastic processes, 2nd ed., Springer, Berlin 2003

[12] I. Karatzas, S. Shreve: Brownian motion and stochastic calculus, Springer, New York 1988

[13] E. H. Lieb, M. Loss: Analysis, 2nd ed., American Mathematical Society, Providence 2001

[14] M. Ondreját: Stochastic nonlinear wave equations in local Sobolev spaces, Electron. J. Probab. 15(2010), 1041-1091

[15] С. Г. Самко, А. А. Килбас, О.И. Маричев: Интегралы и производные дробного порядка и некоторые их приложения, Наука и Техника, Минск 1987 (English translation: S. S. Samko, A. A. Kilbas, O. I. Marichev: Fractional integrals and derivatives, Gordon and Breach, Yverdon 1993)

[16] А. В. Скороход: О существовании и единственности решений стохастических диффузионных уравнений, Сибирск. Мат. Ж. 2(1961), 129-137 (A. V. Skorokhod: On existence and uniqueness of solutions to stochastic diffusion equations, Sibirsk. Mat. Zh. 2(1961), 129-137)

[17] А. В. Скороход: О стохастических дифференциальных уравнениях, Труды VI всесоюзного совещания по теории вероятностей и математической статистике, 159-168, ГИПНЛ 
Литовской ССР, Вильнюс 1962 (А. V. Skorokhod: On stochastic differential equations, Proceedings of the 6th all-union conference on probability theory and mathematical statistics, 159-168, Vil'nyus 1962)

[18] D. W. Stroock, S. R. S. Varadhan: Multidimensional diffusion processes, Springer, Berlin 1979

M.H., Department of Mathematical Analysis, Faculty of Mathematics and Physics, Charles University, Sokolovská 83, 18675 Praha 8, and Institute of Information Theory and Automation of the ASCR, Pod vodárenskou věží 4, 18208 Praha 8, Czech Republic, mar.hofmanova@seznam.cz J.S., Institute of Information Theory and Automation of the ASCR, Pod vodárenskou věží 4, 18208 Praha 8, Czech Republic, seidler@utia.cas.cz 